\title{
Standard forms with two variable zeros and generalized optimal proportional integral derivative (PID) design for all pole systems
}

\author{
Yavuz Sari ${ }^{1}$ and Ali Fuat Boz ${ }^{2 \star}$ \\ ${ }^{1}$ Department of Industrial Electronics, Hendek Vocational High School, Sakarya University, Turkey. \\ ${ }^{2}$ Department of Electronics and Computer Education, Technical Education Faculty, Sakarya University, Turkey.
}

Accepted 21 April, 2011

\begin{abstract}
Other than the classical methods, the use of standard forms for control system design is well known. Since first introduced in 1950s, many new contributions have been proposed in the literature. In these contributions, the standard forms are obtained for all poles and with no zero, one zero and two zeros systems. In this study, for the first time, optimum values of standard form coefficients with five pole and two variable zeros are obtained for the integral squared time error (ISTE) and integral of the squared time error $\left(\mathrm{IST}^{2} \mathrm{E}\right)$ criteria. Again in this study, a generalized controller design approach using standard forms with all pole and two variable zeros has been given for $\mathrm{n}^{\text {th }}$ degree all pole systems. In the proposed approach, a proportional integral derivative (PID) controller in the feed forward path and a polynomial controller, which its degree changes according to system degree in the inner feedback path, have been used. Parameters of these controllers are obtained using standard form coefficients and the proposed simple mathematical operations. Comparative examples for the use of the proposed approach together with some well known methods are also given in the MATLAB.
\end{abstract}

Key words: Standard forms, controller design, proportional integral derivative (PID), optimization.

\section{INTRODUCTION}

Because of the robust performance and easiness of the design steps, the use of classical controllers is still popular despite of many proposed modern controller design methods. One of these classical methods is the optimal controller design method. Main idea of this method is to minimize the error signal by adjusting the controller parameters with respect to the system transfer function. In the literature, many error criteria have been proposed for minimizing the error signal. One of the most popular criteria is the integral squared error (ISE) criterion since these allowed solutions to be obtained in the sdomain by using Parseval's theorem (Chen, 1994). Others are integral absolute error (IAE) and integral time absolute error (ITAE) criteria. But, despite of the ISE criterion, the results of the IAE and ITAE criteria, which can be found in many textbooks, are obtained using extensive computations or by simulations. In the classical

\footnotetext{
*Corresponding author. E-mail: afboz@sakarya.edu.tr. Tel: +90.264 .2956451 . Fax: +90.264.2956424.
}

approach of the optimal controller design method, in every case, this minimization operation must be performed for the system. Thus, this method takes long time and needs expert person. It is therefore not very practical. Graham and Lathrop (1953) introduced a new approach to solve these problems. In this method, closed loop transfer function of the system is introduced as a standard form, which is given in Equation 1. Then, this closed loop transfer function's parameters are obtained by minimizing the error signal of the transfer function with respect to the well known error criteria.

Subsequently, this readily available transfer function can be used to obtain the controller parameters with the uncompensated system transfer function by simply equating these two closed loop transfer function. Thus, the approach eliminates the time consuming optimization procedures and need of the expert person.

$$
\frac{C(s)}{R(s)}=\frac{c_{k} s^{k}+c_{k-1} s^{k-1}+\ldots+c_{1} s+c_{0}}{s^{n}+d_{n-1} s^{n-1}+\ldots+d_{1} s+d_{0}}
$$


Graham and Lathrop (1953) have used the IAE and ITAE criteria for obtaining the standard forms. They have also suggested the optimal standard form coefficients for only all pole standard forms. In 1996, Dorf and Bishop (1996) suggested the ISE criterion, but they did not give the standard form coefficients. Instead, they have obtained the standard form coefficients for the systems with one zero using the ITAE criteria for a ramp input.

Dorf and Bishop (1995, 1996) and some textbooks, devoting a separate section for the subject, suggest and use, $c_{0}=d_{0}$ and $c_{1}=d_{1}$ to have a zero steady-state error for a ramp input for obtaining the closed loop transfer functions of $m$ poles standard forms with one zero. On the other hand, this restricts the independently chosen controller parameters. Also this does not mean that the obtained optimal coefficients are also optimal for the step input signal. If the optimal coefficients of the standard forms with a zero want to be obtained for a step input, then $c_{1} \neq d_{1}$ must be chosen (Atherton and Boz, 1998).

Otherwise, obtained standard forms may cause very oscillatory step responses for the same systems and overshoot of the responses increases. Coefficients of the standard forms with all pole and one zero have been obtained for ISTE and IST ${ }^{2} E$ criteria by A. F. Boz and the results are given in Boz (1999). Recently, for four poles with two zeros systems, optimum values of standard form coefficients have been obtained for the ISTE criterion by Sari and Boz (2009) and for the $I S T^{2} E$ criterion by Boz and Sari (2009a). In 2009, Boz and Sari (2009b) have also suggested a new approach to obtain the parameters of the PI controller, which is used in the feed forward path, and a polynomial controller, which is used in the feedback path, using the standard forms with a zero for all pole systems. In this study, for the first time, optimum values of standard form coefficients with five pole and two variable zeros are obtained for the ISTE and IST ${ }^{2} \mathrm{E}$ criteria.

Again in this study, to show the use of obtained standard form coefficients in the controller design, a new simple generalized controller design approach for the systems with all pole transfer function is introduced. The design approach uses the standard forms with $c_{1} \neq d_{1}$, and $c_{2} \neq d_{2}$ optimized for the ISTE and IST ${ }^{2} E$ criteria. In the proposed approach, a proportional integral derivative (PID) controller, which is in the feed forward and a polynomial controller, which is in the feedback path, are used. Degree of the polynomial controller can be determined according to the degree of the all pole system. Parameters of these controllers are obtained using standard form coefficients and the proposed simple mathematical operations.

\section{MATERIALS AND METHODS}

\section{Integral performance criteria}

A dynamical system's performance is usually defined with its transient response. The transient response of a system for a ramp or a step input is determined by measuring the system's output in terms of rising time, settling time, overshoot and steady-state error. Ideally, output signal of the system must follow the input signal exactly. Thus, ideally, all of these measurements must be zero. On the other hand, in practice, this is impossible; therefore the output must follow the input as close as possible. If the system's performance is not the desired one, then a controller is usually added to the system to achieve the desired responses. In the literature, many different controller structures and design methods can be found. Amongst the popular controller design methods, optimization methods can be counted.

As it was explained before, minimization of the error signal is performed by using a known function, which is called performance index. A performance index consists of some performance characteristics, which the system tries to achieve. This function, depends on the controller parameters, and is optimized numerically. This procedure yields optimal controller parameters and these are also appropriate for the desired system response. Adjusting the performance index to its minimum value by using the system parameters yields the optimal response. Therefore, this type of system is called optimum controller system. The performance index value of a system is always a positive number or zero. Therefore, ideal system is described as a system which minimizes this index.

The controller is normally required to minimize the error signal, which is the difference between reference input $(r(t))$, and controlled output signal $(\mathrm{c}(\mathrm{t}))$ as given in Equation 2

$e(t) \rightarrow 0 \quad t \geq 0$.

Thus, a criterion suitable to characterize the time response of a system is usually given as an integral function of the error, or its weighted products. A general form of an integral error criterion may be represented as follows

$J=\int_{0}^{\infty} \Phi[e(t), t] d t$

Therefore, an optimum dynamic performance may be taken as the time response which gives a minimum value of $\mathrm{J}$. The integral performance criterion can be expressed in different forms thus a control system is considered optimal if the selected performance index is minimized by varying the controller parameters. Since the optimal parameters depend directly on the selected criterion, it is important to reexamine some of the well known integral performance criteria. For over forty years, many approaches have been used for developing design criteria for optimum transient behavior of a system. Two of the most frequently used criteria, which are the ISE and the IAE, were suggested by Graham and Lathrop (1953). The performance indices of the two criteria are given by

$$
\begin{aligned}
J_{I S E} & =\int_{0}^{\infty} e^{2}(t) d t \\
J_{I A E} & =\int_{0}^{\infty}|e(t)| d t .
\end{aligned}
$$

Time weighted versions of these two criteria have also been introduced in Zhuang (1992) for ISE and Graham and Lathrop (1953) for IAE. More general representations of these criteria are

$$
J_{n}(\theta)=\int_{0}^{\infty}\left[t^{n} e(\theta, t)\right]^{2} d t
$$


which is the general time weighted integral squared error criterion, and

$$
J_{n}^{\prime}(\theta)=\int_{0}^{\infty} t^{n}|e(\theta, t)| d t
$$

which is the general time weighted integral absolute error criterion where $\theta$ denotes variable parameters which are chosen to minimize $J_{n}(\theta)$. According to Formula (6), the $J_{0}, J_{1}$ and $J_{2}$ are called ISE, ISTE and IST ${ }^{2} E$, respectively.

Graham and Lathrop (1953) have obtained these optimal transfer functions by using experimental methods in the time domain. These methods also give relatively high error rate and they are difficult to implement. Therefore, there was no interest to this issue until recently. On the other hand, Astrom (1970) proposed an algorithm to calculate the ISE or its derivatives without error for linear systems in 1970.

In the proposed structure,

$$
j_{0}=\int_{0}^{\infty}\left[e^{2}(t)\right] d t
$$

is given in the s domain as follow,

$$
j_{0}=\frac{1}{2 \pi j} \int_{0}^{\infty} E(s) E(-s) d s
$$

where, $E(s)={ }^{A(s)} / B(s)$. Polynomials of $\mathrm{A}(\mathrm{s})$ and $\mathrm{B}(\mathrm{s})$ are also given as follow;

$$
\begin{aligned}
& A(s)=a_{0} s^{m}+a_{1} s^{m-1}+\ldots+a_{m-1} s+a_{m} \\
& B(s)=b_{1} s^{m-1}+b_{2} s^{m-2}+\ldots+b_{m-1} s+b_{m}
\end{aligned}
$$

Again, time-weighted integral squared error criterion, which is given in the form of $j_{n}=\int_{0}^{\infty}\left[t^{n} e(t)\right]^{2} d t$, can be easily calculated in the $\mathrm{s}$ domain using $L[t f(t)]=\frac{-d}{d s} F(s)$ property (Astrom, 1970).

\section{Standard forms}

Today, standard forms take more and more interest by the scientist. Many of the textbooks devote a chapter for this subject as well. Amongst the reasons of this, simple algebraic operations, which give the optimal system coefficients, can be countered.

Generally, the closed loop transfer function of a plant can be represented by

$$
\frac{C(s)}{R(s)}=\frac{c_{k} s^{k}+c_{k-1} s^{k-1}+\ldots+c_{1} s+c_{0}}{s^{n}+d_{n-1} s^{n-1}+\ldots+d_{1} s+d_{0}}
$$

The steady-state error of this system can be shown to be as,

$$
\begin{gathered}
e_{s s}=\left(c_{0}-d_{0}\right) r(t)+\left(c_{1}-d_{1}\right) \frac{d r(t)}{d t}+\left(c_{2}-d_{2}\right) \frac{d^{2} r(t)}{d t^{2}}+\ldots \\
\ldots+\left(c_{k-1}-d_{k-1}\right) \frac{d^{k-1} r(t)}{d t^{k-1}}+\left(c_{k}-1\right) \frac{d^{k} r(t)}{d t^{k}}
\end{gathered}
$$

The form of the input $r(t)$ determines the size of the steady-state error. In order to get zero steady-state error for a step function input, it is required that $c_{0}=d_{0}$. Since the order of the numerator of $\mathrm{C}(\mathrm{s}) / \mathrm{R}(\mathrm{s})$ can be less than or equal to the order of the denominator, there are many possibility of $\mathrm{C}(\mathrm{s}) / \mathrm{R}(\mathrm{s})$ for which the steady-state error is zero with a step input.

On the other hand, steady-state error with a ramp function input only become zero when $c_{0}=d_{0}$ and $c_{1}=d_{1}$. This means that the system is type 2 or higher.

\section{RESULTS}

\section{Standard forms with two zeros}

With two zeros, the transfer function of a standard form may be denoted by $T_{2 j}(s)$ with the denominator given as in the all pole form and the numerator by $c_{2} s^{2}+c_{1} s+1$, which is

$$
T_{2 j}(s)=\frac{c_{2} s^{2}+c_{1} s+1}{s^{j}+d_{j-1} s^{j-1}+\ldots+d_{1} s+1} .
$$

In many cases, contrary to the classical textbooks devoting a chapter for this subject, it is not appropriate to take $c_{1}=d_{1}$, and $c_{2}=d_{2}$ for controller design and minimizing the error index procedures. In the case of $\mathrm{c}_{2} \neq$ $d_{2}$ and $c_{1} \neq d_{1}$, the optimum values of the $d$ coefficients vary with the choice of $c_{1}$ and $c_{2}$. Recently, for four pole with two variable zeros systems, optimum values of standard form coefficients have obtained for the ISTE criterion by Sari and Boz (2009) and for the $I S T^{2} E$ criterion by Boz and Sari (2009a). In this study, optimum values of standard form coefficients with five pole and two variable zeros are obtained for the ISTE and IST ${ }^{2} E$ ) criteria. The optimum values of these coefficients for the $J_{1}$ and $J_{2}$ criteria for $T_{24}(s)$ as a function of $c_{1}$ and $c_{2}$, are given here in Figures 1 and 2, respectively. The optimum values of these coefficients for the $J_{1}$ and $J_{2}$ criteria for $\mathrm{T}_{25}(\mathrm{~s})$ as a function of $\mathrm{c}_{1}$ and $\mathrm{C}_{2}$ are given here in Figures 3 and 4 , respectively.

\section{Optimal controller design approach for $n^{\text {th }}$ degree all pole systems}

Here, a new optimal controller design approach for $n^{\text {th }}$ degree all pole systems has been introduced. In the suggested controller scheme, as shown in Figure 5, a PID controller in the feed forward path and a polynomial controller, which its degree changes according to system degree, in the inner feedback path have been used. In 


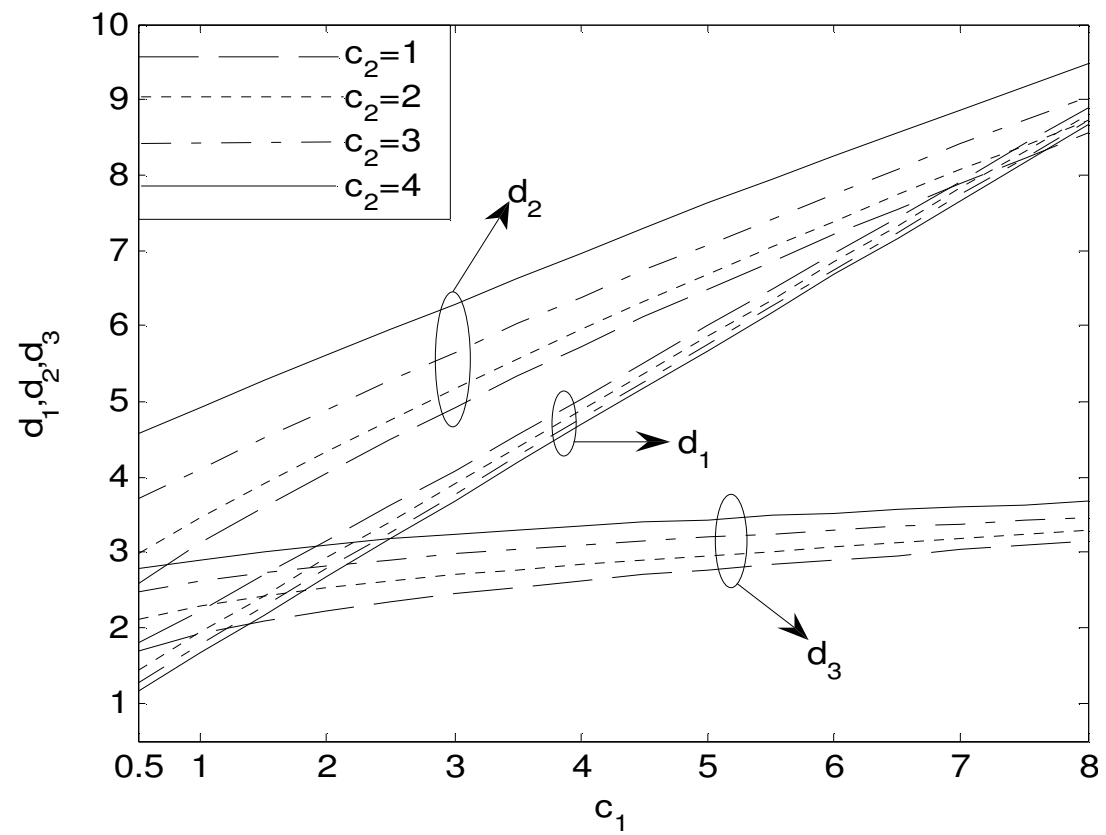

Figure 1. Optimum values of $d_{1}, d_{2}$ and $d_{3}$ for $T_{24}(s)$ with ISTE criterion.

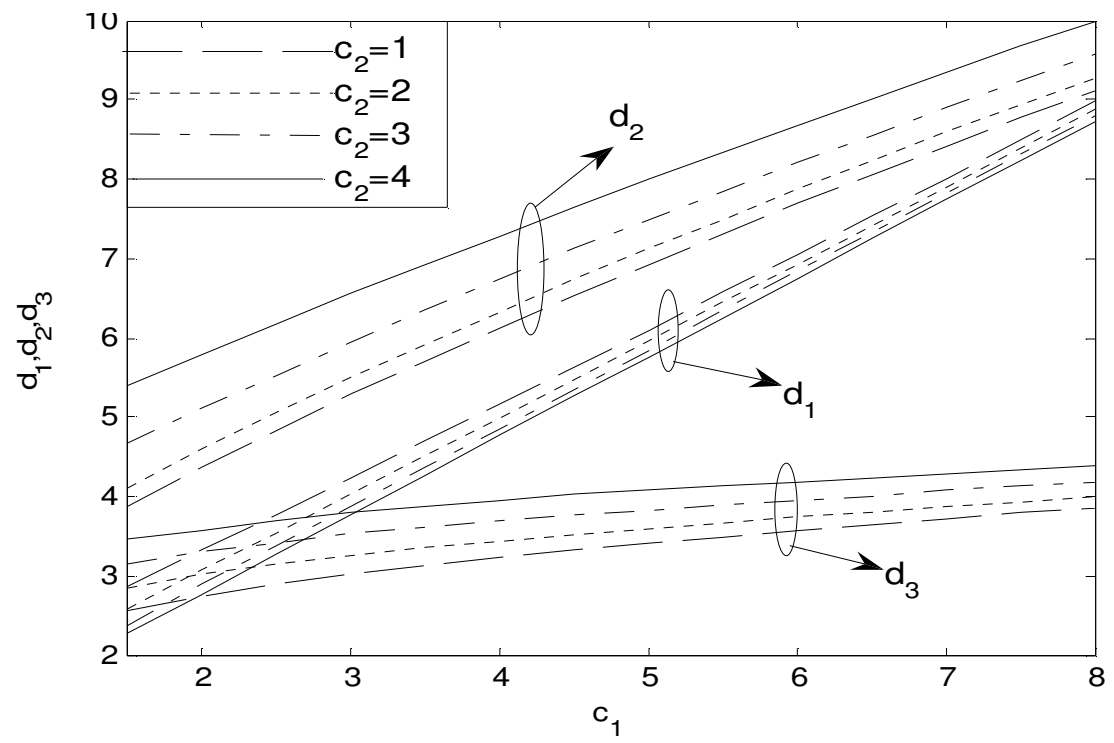

Figure 2. Optimum values of $d_{1}, d_{2}$ and $d_{3}$ for $T_{24}$ (s) with $I S T^{2} E$ criterion.

the suggested approach, generalized formulae for designing optimal controller parameters have been obtained using the standard forms with two zeros. Two comparative examples are given here to show the validity of the method.

\section{Generalized optimal controller design approach for $\mathrm{n}^{\text {th }}$ degree all pole systems}

$\mathrm{n}^{\text {th }}$ degree all pole system's transfer function can be represented by

$G(s)=\frac{a_{0}}{b_{n} s^{n}+b_{n-1} s^{n-1}+\ldots+b_{2} s^{2}+b_{1} s+b_{0}}$

This system can be controlled using a PID controller in the feed forward path and a polynomial controller in the inner feedback path as shown in Figure 5.

Closed loop transfer function of the inner feedback controller and the system can be represented as, 


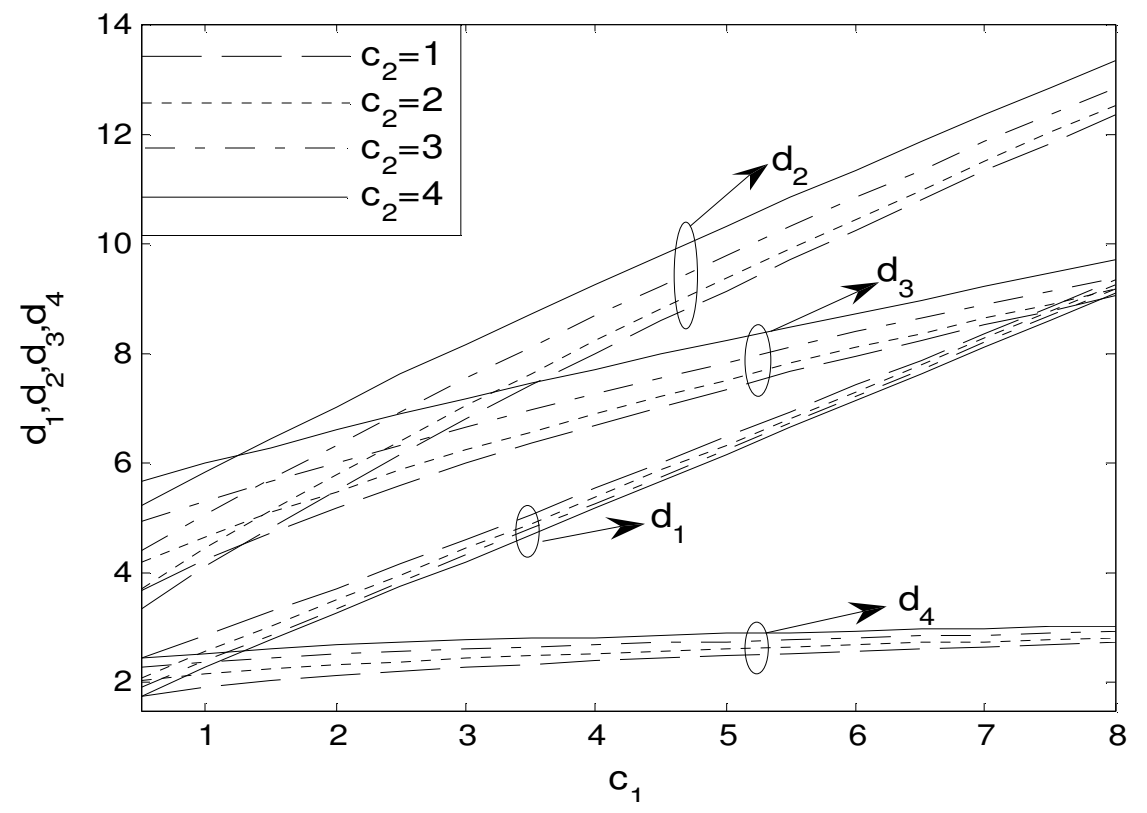

Figure 3. Optimum values of $d_{1}, d_{2}, d_{3}$ and $d_{4}$ for $T_{25}$ (s) with ISTE criterion.

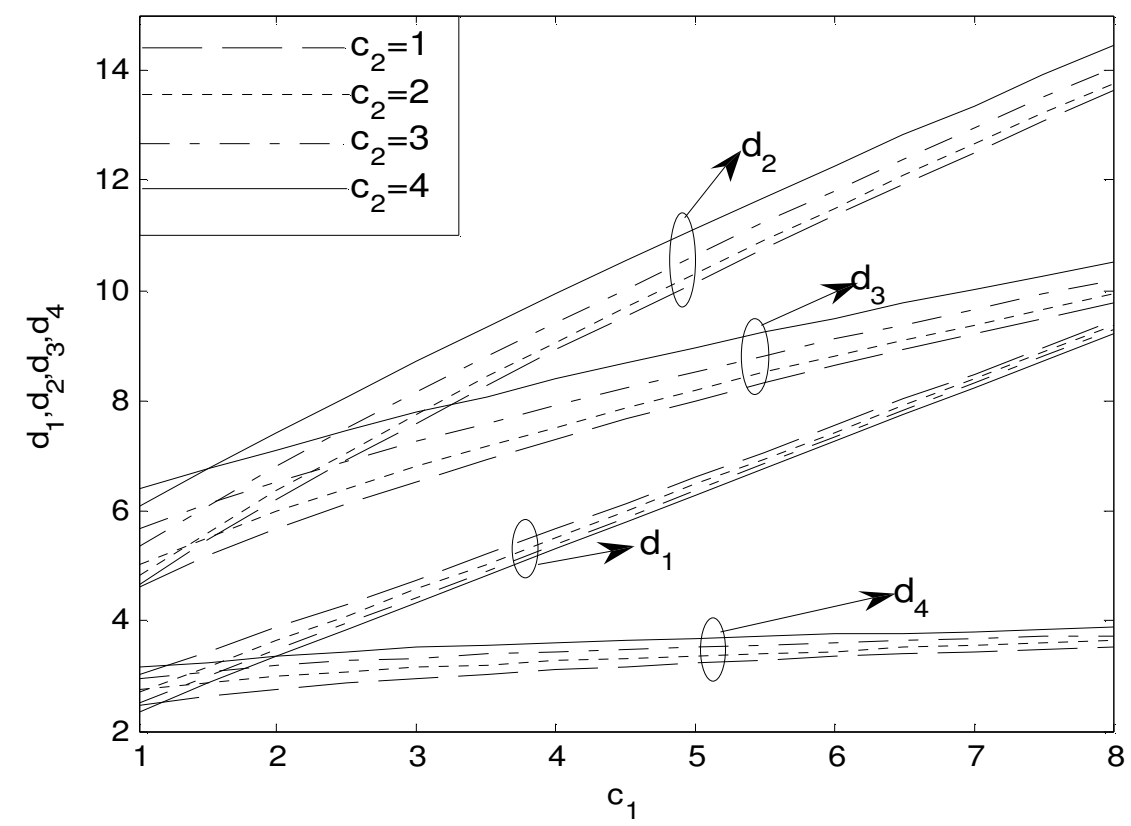

Figure 4. Optimum values of $d_{1}, d_{2}, d_{3}$ and $d_{4}$ for $T_{25}$ (s) with $I S T^{2} E$ criterion.

$$
\begin{gathered}
G(s)=\frac{a_{0}}{b_{n} s^{n}+b_{n-1} s^{n-1}+\left(b_{n-2}+a_{0} k_{n-2}\right) s^{n-2}+\left(b_{n-3}+a_{0} k_{n-3}\right) s^{n-3}+\ldots} \\
. .+\left(b_{1}+a_{0} k_{1}\right) s+a_{0} k_{0}+b_{0}
\end{gathered}
$$

$$
\begin{aligned}
T(s)= & \frac{l_{2} a_{0} s^{2}+l_{1} a_{0} s+l_{0} a_{0}}{b_{n} s^{n+1}+b_{n-1} s^{n}+\left(b_{n-2}+a_{o} k_{n-2}\right) s^{n-1}+\ldots} \\
& \ldots+\left(b_{1}+a_{0} k_{1}+a_{0} l_{2}\right) s^{2}+\left(a_{0} k_{0}+a_{0} l_{1}+b_{0}\right) s+a_{0} l_{0}
\end{aligned}
$$

and the resulting closed loop transfer function of $G^{\prime}(s)$, the PID controller and the unity feedback is given by, which can be normalized to the form 


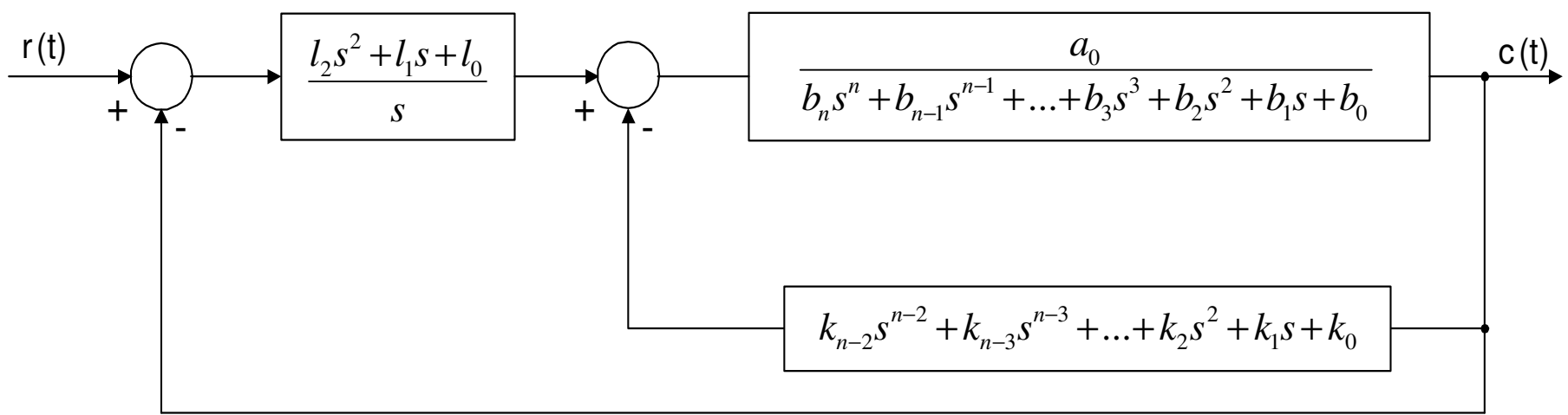

Figure 5. The use of PID controller in the feed forward path for $\mathrm{n}^{\text {th }}$ degree all pole systems.

$$
\begin{gathered}
T\left(s_{m}\right)=\frac{s_{m}^{2} \alpha^{2} \frac{l_{2}}{l_{0}}+s_{m} \alpha^{1}+1}{s_{0}^{n+1}+s_{m}^{n} \alpha^{-1} \frac{b_{m-1}}{b_{n}}+s_{m}^{n-1} \alpha^{-2}\left(\frac{b_{n-2}+a_{b} k_{n-2}}{b_{n}}\right)+s_{m}^{n-2} \alpha^{-3}\left(\frac{b_{n-3}+a_{0} k_{n-3}}{b_{n}}\right)+\ldots} \\
s_{n}^{3} \alpha^{-(n-2)}\left(\frac{b_{2}+a_{0} k_{2}}{b_{n}}\right)+s_{m}^{2} \alpha^{(n-1)}\left(\frac{a_{0} k_{1}+a_{b} l_{2}+b_{1}}{b_{n}}\right)+s_{m} \alpha^{n}\left(\frac{a_{0} k_{0}+a_{0} l_{1}+b_{0}}{b_{n}}\right)+1
\end{gathered}
$$

where $\alpha=\left(\frac{b_{n}}{a_{0} l_{0}}\right)^{-1 / n+1}$ and $s_{m}=s / \alpha=s\left(b_{n} / a_{0} l_{0}\right)^{1 / n+1}$, which means that the response of the system will be faster than the normalized response by a factory of $\alpha$. To simplify the analysis, numerator and denominator coefficients of the normalized system's closed loop transfer function can be arranged as,

$$
\begin{aligned}
& d_{n}=\alpha^{-1} \frac{b_{n-1}}{b_{n}} \\
& d_{n-1}=\alpha^{-2}\left(\frac{b_{n-2}+a_{0} k_{n-2}}{b_{n}}\right) \\
& d_{n-2}=\alpha^{-3}\left(\frac{b_{n-3}+a_{0} k_{n-3}}{b_{n}}\right) \\
& d_{3}=\alpha^{-(n-2)}\left(\frac{b_{2}+a_{0} k_{2}}{b_{n}}\right) \\
& d_{2}=\alpha^{-(n-1)}\left(\frac{a_{0} k_{1}+a_{0} l_{2}+b_{1}}{b_{n}}\right) \\
& d_{1}=\alpha^{-n}\left(\frac{a_{0} k_{0}+a_{0} l_{1}+b_{0}}{b_{n}}\right) \\
& c_{1}=\alpha \frac{l_{1}}{l_{0}}
\end{aligned}
$$

Substituting these values into the Equations (18) gives the new transfer function of the system, to be

$$
T_{2(n+1)}\left(s_{m}\right)=\frac{c_{2} s_{m}^{2}+c_{1} s_{m}+1}{s_{m}^{n+1}+d_{n} s_{m}^{n}+d_{n-1} s_{m}^{n-1}+\ldots+d_{2} s_{m}^{2}+d_{1} s_{m}+1}
$$

$\mathrm{n}+1$ degree standard form with two variable zero can be represented as in Equation (27). Using Equations (19) to (26) with the transfer function given in Equation (27) the controller parameters results as,

$$
\begin{aligned}
& l_{0}=\alpha^{n+1} \frac{b_{n}}{a_{0}}=\frac{b_{n-1}^{n+1}}{a_{0} b_{n}^{n}} \frac{1}{d_{n}^{n+1}} \\
& l_{1}=\alpha^{n} c_{1} \frac{b_{n}}{a_{0}}=\frac{b_{n-1}^{n}}{a_{0} b_{n}^{n-1}} \frac{c_{1}}{d_{n}^{n}} \\
& l_{2}=\alpha^{n-1} \frac{c_{2} b_{n}}{a_{0}}=\frac{b_{n-1}^{n-1}}{a_{0} b_{n}^{n-2}} \frac{c_{2}}{d_{n}^{n-1}}
\end{aligned}
$$

$$
k_{0}=\alpha^{n} \frac{\left(d_{1}-c_{1}\right) b_{n}}{a_{0}}-\frac{b_{0}}{a_{0}}=\frac{b_{n-1}^{n}}{a_{0} b_{n}^{n-1}} \frac{\left(d_{1}-c_{1}\right)}{d_{n}^{n}}-\frac{b_{0}}{a_{0}}
$$

$$
k_{1}=\alpha^{n-1} \frac{\left(d_{2}-c_{2}\right) b_{n}}{a_{0}}-\frac{b_{1}}{a_{0}}=\frac{b_{n-1}^{n-1}}{a_{0} b_{n}^{n-2}} \frac{\left(d_{2}-c_{2}\right)}{d_{n}^{n-1}}-\frac{b_{1}}{a_{0}}
$$

$$
k_{2}=\alpha^{n-2} \frac{d_{3} b_{n}}{a_{0}}-\frac{b_{2}}{a_{0}}=\frac{b_{n-1}^{n-2}}{a_{0} b_{n}^{n-3}} \frac{d_{3}}{d_{n}^{n-2}}-\frac{b_{2}}{a_{0}}
$$


Table 1. Suggested controller design method's results for Example 1.

\begin{tabular}{ccc}
\hline & PID IST $^{2} \mathbf{E}$ & PID ISTE \\
\hline $\mathrm{c}_{1}$ & 6 & 6 \\
$\mathrm{c}_{2}$ & 4 & 4 \\
$\mathrm{~d}_{1}$ & 6.747 & 6.671 \\
$\mathrm{~d}_{2}$ & 8.68 & 8.26 \\
$\mathrm{~d}_{3}$ & 4.188 & 3.523 \\
& & \\
$\mathrm{l}_{2}$ & 1.9006 & 2.6855 \\
$\mathrm{l}_{1}$ & 3.4037 & 5.717 \\
$\mathrm{l}_{0}$ & 0.6773 & 1.3523 \\
$\mathrm{k}_{1}$ & 1.557 & 2.1904 \\
$\mathrm{k}_{0}$ & -0.5764 & -0.3607 \\
\hline
\end{tabular}

Table 2. Controller parameters, which are obtained using the R.Z-N, A-H and Gain-phase methods for Example 1.

\begin{tabular}{cccc}
\hline & R. Z-N & A-H & Gain-phase \\
\hline $\mathrm{K}_{\mathrm{c}}$ & 2.334 & 2.334 & 2.334 \\
$\omega_{\mathrm{c}}$ & 1.4144 & 1.4144 & 1.4144 \\
$\mathrm{~T}_{\mathrm{c}}$ & 4.4423 & 4.4423 & 4.4423 \\
$\alpha$ & & 4 & \\
$\phi_{m}$ & & 45 & \\
$\beta$ & & & \\
$\mathrm{K}_{\mathrm{p}}$ & 0.7307 & & \\
$\mathrm{~T}_{\mathrm{i}}$ & 1.4004 & 1.6504 & 1.1882 \\
$\mathrm{~T}_{\mathrm{d}}$ & 2.2212 & 3.4138 & 1.961 \\
\hline
\end{tabular}

$$
\begin{aligned}
& k_{3}=\alpha^{n-3} \frac{d_{4} b_{n}}{a_{0}}-\frac{b_{3}}{a_{0}}=\frac{b_{n-1}^{n-3}}{a_{0} b_{n}^{n-4}} \frac{d_{4}}{d_{n}^{n-3}}-\frac{b_{3}}{a_{0}} \\
& k_{n-3}=\alpha^{3} \frac{d_{n-2} b_{n}}{a_{0}}-\frac{b_{n-3}}{a_{0}}=\frac{b_{n-1}^{3}}{a_{0} b_{n}^{2}} \frac{d_{n-2}}{d_{n}^{3}}-\frac{b_{n-3}}{a_{0}} \\
& k_{n-2}=\alpha^{2} \frac{d_{n-1} b_{n}}{a_{0}}-\frac{b_{n-2}}{a_{0}}=\frac{b_{n-1}^{2}}{a_{0} b_{n}} \frac{d_{n-1}}{d_{n}^{2}}-\frac{b_{n-2}}{a_{0}}
\end{aligned}
$$

or generalizing the formula for $k=0,1,2,3,4, \ldots . n-2$,

$$
\begin{aligned}
\sum_{i=0}^{n-2} k_{i} & =\sum_{i=0}^{1}\left(\alpha^{n-i} \frac{\left(d_{i+1}-c_{i+1}\right) b_{n}}{a_{0}}-\frac{b_{i}}{a_{0}}\right)+\sum_{i=2}^{n-2}\left(\alpha^{n-i} \frac{d_{i+1} b_{n}}{a_{0}}-\frac{b_{i}}{a_{0}}\right) \\
\sum_{i=0}^{2} l_{i} & =\sum_{i=0}^{2} \alpha^{n-i+1} c_{i} \frac{b_{n}}{a_{0}}
\end{aligned}
$$

and

$\alpha=\frac{b_{n-1}}{b_{n}} \frac{1}{d_{n}}$

can be obtained.

\section{DISCUSSION}

Here, advantages and validity of the proposed approach over some well known design methods are given with two different examples for comparison.

\section{Example 1}

Consider the third order transfer function,

$G(s)=\frac{3}{s^{3}+5 s^{2}+2 s+3}$.

Comparing the transfer function of this system with Equation 14, gives the following values, $n=3, a_{0}=3, b_{3}=$ $1, b_{2}=5, b_{1}=2$ and $b_{0}=3$. Then choosing $c_{1}=6$ and $c_{2}=$ 4 for ISTE and IST ${ }^{2} E$ criteria from Figures 1 and 2 and using them in the generalized formulae, which are given in Equations 36 and 37, result in the controller parameters and these data are summarized in Table 1. For the same system, results of some well known PID controller design methods are also obtained. These are refined Ziegler-Nichols (R.Z-N) (Hang et al., 1991), Astrom Hagglund (A-H) (Astrom and Hagglund, 1984) and Gain-phase (Zhuang and Atherton, 1993) controller design methods. Summary of the results obtained from these methods are given in Table 2. Finally, step responses of all design methods together with that of the suggested design method are plotted in the same figure for comparison (Figure 6).

It is seen from Figure 6 that, the Gain-phase method gives most oscillatory response and longest settling time together with the A-H method. On the other hand, result of the suggested design approach for $I S T^{2} E$ gives minimum overshoot, settling time and little oscillation. ISTE design also gives relatively less overshoot and short settling time but its response is faster than the $I S T^{2} E$ design.

\section{Example 2}

In this case, consider the fourth order transfer function,

$G(s)=\frac{5}{s^{4}+5 s^{3}+9 s^{2}+15 s+7}$

Coefficients of the transfer function are $n=4, a_{0}=5, b_{4}=$ $1, b_{3}=5, b_{2}=9, b_{1}=15$ and $b_{0}=7$. Again, choosing $c_{1}=6$ 


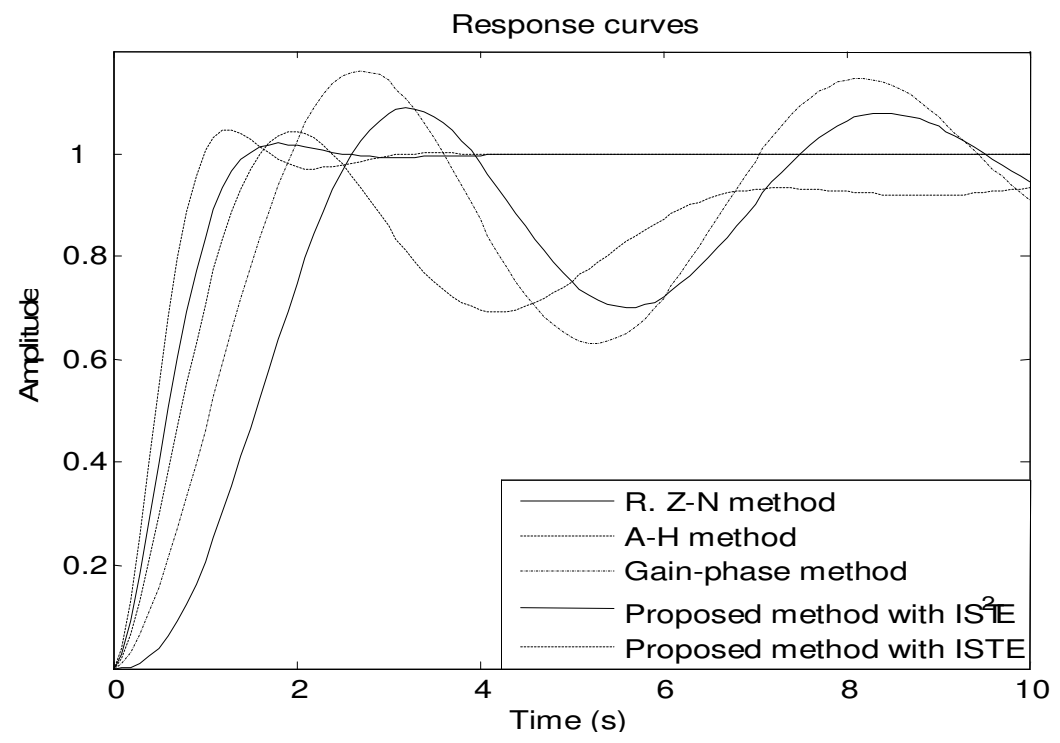

Figure 6. Step responses for Example 1.

Table 3. Suggested controller design method's results for Example 2.

\begin{tabular}{ccc}
\hline & PID IST $^{2}$ E & PID ISTE \\
\hline $\mathrm{c}_{1}$ & 6 & 6 \\
$\mathrm{c}_{2}$ & 4 & 4 \\
$\mathrm{~d}_{1}$ & 7.2598 & 7.1453 \\
$\mathrm{~d}_{2}$ & 12.2592 & 11.3414 \\
$\mathrm{~d}_{3}$ & 9.5002 & 8.746 \\
$\mathrm{~d}_{4}$ & 3.754 & 2.947 \\
& & \\
$\mathrm{l}_{2}$ & 1.8902 & 3.9071 \\
$\mathrm{l}_{1}$ & 3.7765 & 9.9435 \\
$\mathrm{l}_{0}$ & 0.8383 & 2.8118 \\
$\mathrm{k}_{2}$ & 1.5707 & 3.2352 \\
$\mathrm{k}_{1}$ & 0.903 & 4.171 \\
$\mathrm{k}_{0}$ & -0.6071 & 0.498 \\
\hline
\end{tabular}

Table 4. Controller parameters, which are obtained using the R.Z-N, A-H and Gain-phase methods for Example 2.

\begin{tabular}{cccc}
\hline & R. Z-N & A-H & Gain-phase \\
\hline $\mathrm{K}_{\mathrm{c}}$ & 2.2047 & 2.2047 & 2.2047 \\
$\omega_{\mathrm{c}}$ & 1.7318 & 1.7318 & 1.7318 \\
$\mathrm{~T}_{\mathrm{c}}$ & 3.6281 & 3.6281 & 3.6281 \\
$\alpha$ & & 4 & \\
$\phi_{m}$ & & 45 & \\
$\beta$ & & & \\
$\mathrm{K}_{\mathrm{p}}$ & 0.9468 & & \\
$\mathrm{~T}_{\mathrm{i}}$ & 1.3228 & 1.559 & 1.1218 \\
$\mathrm{~T}_{\mathrm{d}}$ & 0.8141 & 2.788 & 1.5292 \\
\hline
\end{tabular}

and $c_{2}=4$ for ISTE and IST ${ }^{2} E$ criteria from the Figures 3 and 4 gives the $d$ coefficients as seen in Table 3 . Using these coefficients in the Equations 36 and 37, result the suggested controller parameters, which are summarized in Table 3. For the same system, the R.Z-N, A-H and Gain-phase methods yield the controller parameters, which are given in Table 4. Step responses of the all design methods are also given in Figure 7.

As can be seen from Figure 7, R. Z-N method gives biggest overshoot. Again R. Z-N, A-H and Gain-phase methods give sluggish responses. On the other hand, $I S T^{2} E$ method provides minimum overshoot and also the settling time of the $\mathrm{IST}^{2} \mathrm{E}$ method is better than that of the other methods. ISTE method also gives relatively fast response with little oscillation.

\section{Conclusion}

In this work, a new approach to obtain the optimal PID and polynomial controller parameters for the $\mathrm{n}^{\text {th }}$ degree all pole system has been introduced. The suggested approach basically uses the optimized standard forms with two variable zeros. Also the approach directly targets the step response shaping in the time domain. It is shown in the work that, in the contrary of the literature, choosing $c_{2} \neq d_{2}, c_{1} \neq d_{1}$ in the standard forms yields relatively better system performances for the step input. It is also generalized formulae for the optimal controller design is suggested in the work, thus the design procedure is simplified and also the need of expert person in controller design is eliminated. Again the suggested approach introduces simple algebraic operations, thus there is no need to optimize the system every time. Therefore, design time is also shortened. As it was shown in the examples, the approach gives better responses over 


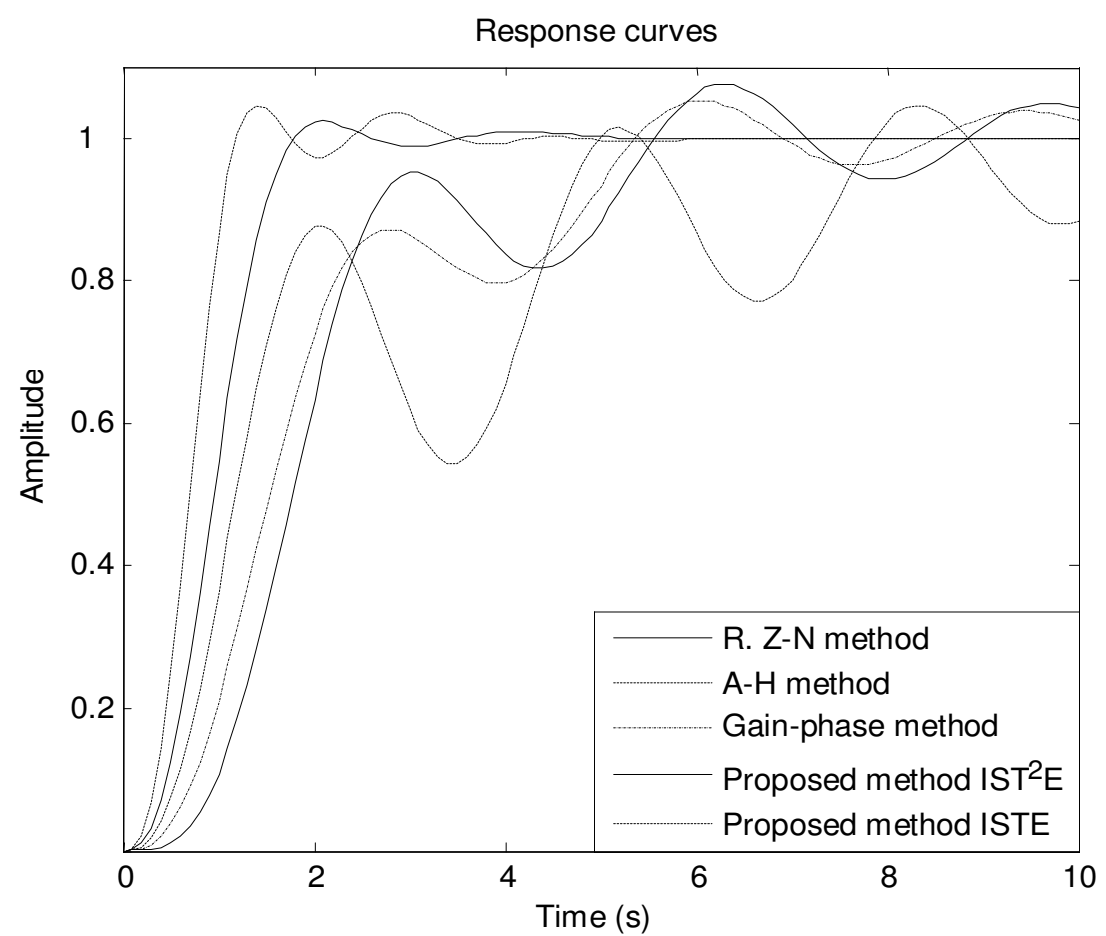

Figure 7. Step responses for Example 2.

some well known design methods, thus it can be preferable in designing the optimum controller. The results of the work can also be used in the state feedback. Addition of these, the work also introduces optimum values of standard form coefficients with five pole and two variable zeros for the ISTE and IST ${ }^{2} E$ criteria.

\section{REFERENCES}

Astrom KJ (1970). Introduction to stochastic control theory. Academic Press, New York.

Astrom KJ, Hagglund T (1984). Automatic Tuning of Simple Regulators with Specification on Phase and Amplitude Margins. Automatica. 20(5): 645-651.

Atherton DP, Boz AF (1998). Using standard forms for controller design, UKACC Int. Conference on Control'98 held at Swansea, UK, pp. 1066-1071.

Boz AF (1999). Computational Approaches to and Comparisons of Design Methods for Linear Controllers. PhD dissertation, Dept. Elect. Eng., University of Sussex, Brighton, UK, pp. 58-85.

Boz AF, Sari Y (2009a). Standard Forms with Two Zeros and Optimal PID-PD Controller Design. 5. International Advanced Technologies Symposium, (IATS'09) held at Karabuk, Turkey, pp. 480-484.
Boz AF, Sari Y (2009b). Generalized Optimal Controller Design for All Pole Systems Using Standard Forms. Sci. Res. Essay., 4(3): 167174.

Chen CT (1994). System and Signal Analysis. $2^{\text {nd }}$ Edition, Saunders College Publishing. Orlando, Florida, USA, pp. 320-321.

Dorf RC, Bishop RH (1995). Modern Control Systems. $7^{\text {th }}$ Edition, Addison-Wesley, Reading, MA, pp. 240-248.

Dorf RC, Bishop RH (1996). Design Using Performance Indices. The Control Handbook. CRC Press, pp. 169-173.

Graham D, Lathrop RC (1953). The Synthesis of Optimum Response: Criteria and Standard Forms. Transact. AIEE., 72: 273-288.

Hang CC, Astrom KJ, Ho WK (1991). Refinements of the ZieglerNichols Tuning Formula, IEE Proceedings-D, 138(2): 111-118.

Sari Y, Boz AF (2009). Autotuning of Second Order Plus Dead Time Systems Using Standard Forms and PID-PD. 5. International Advanced Technologies Symposium, (IATS'09) held at Karabuk, Turkey, pp. 202-206.

Zhuang M (1992). Computer Aided PID Controller Design. PhD dissertation, Dept. of Elect. Eng., University of Sussex, Brighton, UK, pp. 65-102.

Zhuang M, Atherton DP (1993). Automatic Tuning of Optimum PID Controllers. IEE Proceedings-D. 140(3): 216-224. 